\title{
PROPER COVERS FOR LEFT AMPLE SEMIGROUPS
}

\author{
Abdulsalaam El Qallali \\ Department of Mathematics, \\ Al-Fateh University \\ Tripoli, Libya
}

\author{
John Fountain \\ Department of Mathematics, \\ University of York, \\ Heslington, York YO10 5DD, U.K. \\ e-mail : jbf1@york.ac.uk
}

Abstract. A left ample semigroup is a semigroup with a unary operation ${ }^{+}$ which has a $(2,1)$-algebra embedding into a symmetric inverse monoid $I(X)$, the operation ${ }^{+}$on $I(X)$ being defined by $\alpha^{+}=\alpha \alpha^{-1}$. We consider some analogues for left ample semigroups of results on $E$-unitary covers of inverse semigroups due to McAlister and Reilly. The analogue of an E-unitary cover is a proper cover, and we discuss the construction of proper covers in terms of relational homomorphisms, and of dual prehomomorphisms. We observe that our construction gives an $E$-dense proper cover for an $E$-dense left ample semigroup. We also consider proper covers constructed from strict embeddings into factorisable left ample monoids. In contrast to the inverse case, not all proper covers arise in this way. However, in the $E$-dense case, we characterise those $E$-dense proper covers which can be constructed from such embeddings.

\section{INTRODUCTION}

On an inverse semigroup $S$, we define a unary operation ${ }^{+}$by $a^{+}=a a^{-1}$ for $a \in S$ thus making $S$ into a $(2,1)$-algebra. Of course, $S$ also has the unary operation $^{-1}$, but this will not play a significant role in the paper.

A left ample semigroup can now be defined to be a semigroup $S$ with a unary operation ${ }^{+}$such that there is a $(2,1)$-algebra embedding of $S$ into an inverse monoid. As usual, $E(S)$ denotes the set of idempotents of $S$. It is immediate from the definition that, in a left ample semigroup, the idempotents commute with each other, and so $E(S)$ is a subsemilattice of $S$. It is also clear that every inverse semigroup is a left ample semigroup with the operation ${ }^{+}$defined as above.

Since left ample semigroups are $(2,1)$-algebras, the homomorphisms and subalgebras etc. with which we shall be most concerned are those associated with the class of $(2,1)$-algebras. To emphasise this point we shall sometimes use the terms +-homomorphism and +-subsemigroup, etc. In the case of inverse semigroups, all semigroup homomorphisms are +-homomorphisms, and inverse subsemigroups

Date: August 30, 2005.

2000 Mathematics Subject Classification. 20M10.

Key words and phrases. left ample semigroup, proper cover, relational morphism, factorisable semigroup. 
are +-subsemigroups. We shall also use the following notation: if $A$ is a subset of a left ample semigroup $S$, then $A^{+}$denotes the set $\left\{a^{+}: a \in A\right\}$.

On a left ample semigroup $S$, there is a least congruence $\sigma$ such that $S / \sigma$ is a right cancellative monoid. We say that $S$ is proper if for all elements $a$ and $b$ of $S$ such that $a^{+}=b^{+}$and $a \sigma b$, we have $a=b$. It is well known that an inverse semigroup is proper if and only if it is E-unitary (see, for example, [7, Proposition 5.9.1]). Example 3 of [3] shows that the corresponding statement does not hold for left ample semigroups.

Let $S$ be a left ample semigroup and $T$ be a right cancellative monoid. We say that a left ample semigroup $P$ is a proper cover of $S$ (over $T$ ) if $P$ is proper and there is a surjective +-homomorphism $\alpha$ from $P$ onto $S$ which maps $E(P)$ isomorphically onto $E(S)$ (and is such that $P / \sigma \cong T$ ). The existence of proper covers for right ample monoids was established in [3]; the result is easily extended to the semigroup case, and of course, the left ample results are simply the duals. These results are analogues of the corresponding well known covering theorem for inverse semigroups (see, for example [7, Section 5.9] or [9, Section 2.2]).

We relate proper covers to certain relational morphisms in Section 1. We follow this in Section 2 by first recalling the notion of a weakly left ample semigroup, and then constructing such a semigroup $C(S)$ from a left ample semigroup $S$. The elements of $C(S)$ are certain order ideals of $S$ which we call compatible order ideals. This is an analogue (but not a generalisation) of a construction in inverse semigroup theory. In Section 3, we use $C(S)$ to obtain an analogue of one of the results of McAlister and Reilly, namely we show that proper covers of $S$ over a right cancellative monoid $T$ all arise from and give rise to a dual prehomomomorphism from $T$ into $C(S)$.

An inverse monoid $F$ with group of units $G$ is factorisable if every element of $F$ can be written as a product of a unit and an idempotent. An embedding $\varphi$ of an inverse semigroup $S$ into a factorisable inverse monoid $F$ is strict if for each $g \in G$, there is an element $s$ of $S$ such that $s \varphi \leqslant g$. McAlister and Reilly [12] showed that such an embedding gives rise to an $E$-unitary cover of $S$ over $G$, and conversely that every $E$-unitary cover is isomorphic to one constructed in this way. The situation is more complicated in the case of left ample semigroups. We recall the notion of factorisable left ample monoid in Section 4 and point out that every left ample semigroup can be strictly embedded in such a monoid. It is straightforward to show that such embeddings give rise to proper covers. However, the converse is not true as we see in the final section, which is devoted to $E$-dense left ample semigroups. The existence of proper $E$-dense left ample covers for such semigroups was noted in [5]. We show that an $E$-dense left ample semigroup $S$ can be strictly embedded in a factorisable inverse monoid, and that the proper cover that arises from such an embedding is $E$-dense. Not every $E$-dense proper cover arises from a strict embedding into a factorisable inverse monoid, and we intoduce the concept of an even cover to characterise those which do so arise. 


\section{Relational Morphisms, Prehomomorphisms and Proper Covers}

We start with some elementary properties of left ample semigroups which will be used without further mention. Proofs can be found in [4] or [6]

Lemma 1.1. Let $S$ be a left ample semigroup. If $a, b, c \in S$, then

(1) $\left(a^{+} b\right)^{+}=a^{+} b^{+}$,

(2) $a^{+} a=a$,

(3) $a c=b c$ implies $a c^{+}=b c^{+}$,

(4) $a^{+}=(a b c)^{+}$implies $a^{+}=(a b)^{+}$,

(5) $a \sigma b$ if and only if ea $=e b$ for some idempotent $e$.

A relational morphism $\tau: S \rightarrow T$ of left ample semigroups $S$ and $T$ is a relational morphism of $(2,1)$-algebras, that is, $\tau$ maps $S$ to $2^{T}$ and, for all $s, s_{1}, s_{2} \in S$, we have

(1) $s \tau \neq \emptyset$,

(2) $(s \tau)^{+} \subseteq s^{+} \tau$,

(3) $\left(s_{1} \tau\right)\left(s_{2} \tau\right) \subseteq\left(s_{1} s_{2}\right) \tau$.

If $S$ and $T$ are monoids, we also require

(4) $1 \in 1 \tau$.

These conditions ensure that the graph of $\tau$, which we denote by $\operatorname{gr}(\tau)$, is a $(2,1)$ subalgebra of the direct product $S \times T$, and that the projection of $\operatorname{gr}(\tau)$ to $S$ is surjective. In particular, $\operatorname{gr}(\tau)$ is a left ample subsemigroup of $S \times T$.

If $S$ and $T$ are inverse semigroups, then we require

(5) $(s \tau)^{-1}=s^{-1} \tau$

where $X^{-1}=\left\{x^{-1}: x \in X\right\}$ for $X \subseteq T$.

Notice that (5) and (3) together give (2).

We say that $\tau$ is surjective if the projection to $T$ is surjective. In this case, $\operatorname{gr}(\tau)$ is a subdirect product of $S$ and $T$; moreover, we also have a surjective relational morphism $\tau^{-1}: T \leftrightarrow S$ given by $t \tau^{-1}=\{s \in S: t \in s \tau\}$, and, clearly, $\left(\tau^{-1}\right)^{-1}=\tau$.

Examples of relational morphisms between left ample semigroups are given by +-homomorphisms, and inverses of surjective +-homomorphisms. It is easy to verify that composing relational morphisms gives a relational morphism. For example, if $R, S, T$ are left ample semigroups and $\alpha: R \rightarrow S, \beta: R \rightarrow T$ are +-homomorphisms with $\alpha$ surjective, then the composite $\alpha^{-1} \beta$ is a relational morphism from $S$ to $T$. In fact, all relational morphisms arise in this way, for given $\tau: S \rightarrow T$, we may take $R$ to be $\operatorname{gr}(\tau)$, and $\alpha$ and $\beta$ to be the projections to $S$ and $T$ respectively; then $\tau=\alpha^{-1} \beta$.

Let $S$ be a left ample semigroup and $T$ be a right cancellative monoid. We note that $1_{T} \in e \tau$ for every idempotent $e$ of $S$. For, e $e \tau$ is not empty, and if $t \in e \tau$, then $1_{T}=t^{+} \in(e \tau)^{+} \subseteq e \tau$. We say that a surjective relational morphism 
$\tau: S \rightarrow T$ is (left) proper if for all $a, b \in S$,

$$
a \tau \cap b \tau \neq \emptyset \text { implies } a^{+} b=b^{+} a .
$$

We remark that a proper relational morphism $\tau: S \rightarrow T$ is idempotent pure in the sense that if $1_{T} \in a \tau$, then $a$ is idempotent. For, we also have $1_{T} \in a^{+} \tau$ and so we get $\left(a^{+}\right)^{+} a=a^{+} a^{+}$, that is, $a=a^{+}$. When $S$ is inverse and $T$ is a group, being proper is equivalent to being idempotent pure as shown in the next lemma.

Lemma 1.2. Let $\tau: S \multimap G$ be a relational morphism from an inverse semigroup to a group $G$. Then $\tau$ is proper if and only if it is idempotent pure.

Proof. We have already noted that if $\tau$ is proper, then it is idempotent pure. For the converse, suppose that $\tau$ is idempotent pure, that $a, b \in S$ and that $a \tau \cap b \tau \neq \emptyset$. Let $g \in a \tau \cap b \tau$. Then $g^{-1} \in(a \tau)^{-1} \cap(b \tau)^{-1}=a^{-1} \tau \cap b^{-1} \tau$ and hence $1=g^{-1} g \in\left(a^{-1} \tau\right)(b \tau) \subseteq\left(a^{-1} b\right) \tau$. Similarly, 1 is in $\left(a b^{-1}\right) \tau$ so that $a^{-1} b$ and $a b^{-1}$ are idempotent. By [9, Lemma 1.4.12], we have $a a^{-1} b=b b^{-1} a$ and $a b^{-1} b=b a^{-1} a$ so that $\tau$ is left and right proper.

We now show how relational morphisms are related to proper covers. Let the left ample semigroup $P$ be a proper cover of the left ample semigroup $S$ over a right cancellative monoid $T$. Then we have surjective homomorphisms $\alpha$ and $\beta$ :

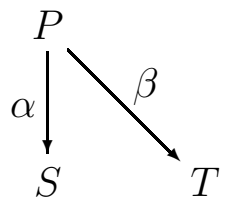

where the restriction of $\alpha$ to $E(P)$ is an isomorphism from $E(P)$ onto $E(S)$, and $\beta \beta^{-1}=\sigma$, the least right cancellative congruence on $P$. The condition on $\alpha$ is equivalent to $\alpha$ being idempotent separating.

For emphasis, we record the following elementary fact as a lemma.

Lemma 1.3. If $p, q \in P$ are such that $p \alpha=q \alpha$ and $p \beta=q \beta$, then $p=q$.

Proof. We have $p^{+}=q^{+}$since $\alpha$ is an idempotent separating +-homomorphism. Also $p \beta=q \beta$ gives $p \sigma q$, and hence $p=q$ since $P$ is proper.

Proposition 1.4. Let $P$ be a proper cover of $S$ over $T$ as above. Then the relational morphism $\tau=\alpha^{-1} \beta$ is proper and $P \cong \operatorname{gr}(\tau)$.

Conversely, if $\tau: S \rightarrow T$ is a proper surjective relational morphism, then $\operatorname{gr}(\tau)$ is a proper cover of $S$ over $T$.

Proof. Let $P$ be a proper cover. Then the mapping $\theta: P \rightarrow \operatorname{gr}(\tau)$ given by $p \theta=(p \alpha, p \beta)$ is clearly a surjective +-homomorphism. By Lemma 1.3, it is injective, and thus $P \cong \operatorname{gr}(\tau)$.

To see that $\tau$ is proper, suppose that $a \tau \cap b \tau \neq \emptyset$, for some $a, b \in S$. Then there are elements $p, q \in P$ such that $p \alpha=a, q \alpha=b$ and $p \beta=q \beta$. Thus $p \sigma q$ 
so that $q^{+} p \sigma p^{+} q$. But $\left(q^{+} p\right)^{+}=q^{+} p^{+}=\left(p^{+} q\right)^{+}$, so that $q^{+} p=p^{+} q$ since $P$ is proper. Applying $\alpha$ gives $b^{+} a=a^{+} b$.

For the converse, suppose that $\tau: S \rightarrow T$ is a surjective proper relational morphism, and let $\alpha: \operatorname{gr}(\tau) \rightarrow S, \beta: \operatorname{gr}(\tau) \rightarrow T$ be the projections. Since $E(\operatorname{gr}(\tau))=\{(e, 1): e \in E(S)\}$, it is clear that $\alpha$ is idempotent separating.

Since $T$ is right cancellative, so is $\operatorname{Im} \beta$, and so we have $\sigma \subseteq \beta^{-1} \beta$. Conversely, if $(a, t),(b, u) \in \operatorname{gr}(\tau)$ and $(a, t) \beta=(b, u) \beta$, then $t=u$ so that $a \tau \cap b \tau \neq \emptyset$, and hence $a^{+} b=b^{+} a$. Thus $\left(a^{+} b^{+}, 1\right)(a, t)=\left(a^{+} b^{+}, 1\right)(b, u)$ so that $(a, t) \sigma(b, u)$ and so $\sigma=\beta^{-1} \beta$.

Now let $(a, t),(b, u) \in \operatorname{gr}(\tau)$ be such that $(a, t)^{+}=(b, u)^{+}$and $(a, t) \sigma(b, u)$, that is, $\left(a^{+}, 1\right)=\left(b^{+}, 1\right)$ and $t=(a, t) \beta=(b, u) \beta=u$. As in the preceding paragraph, $a^{+} b=b^{+} a$, and since $a^{+}=b^{+}$, we have $a=b$, so that $(a, t)=(b, u)$ and $\operatorname{gr}(\tau)$ is proper.

It follows from the proposition that finding all proper covers of a left ample semigroup $S$ over a right cancellative monoid $T$ is equivalent to finding all surjective proper relational morphisms $S \rightarrow T$, or to finding all surjective relational morphisms $\rho: T \nrightarrow S$ which satisfy:

$$
a, b \in t \rho \text { implies } a^{+} b=b^{+} a .
$$

Such relational morphisms $\rho$ will also be called proper.

This parallels the case for certain covers of regular semigroups [1] and $E$-unitary covers of inverse semigroups [12]. In these cases, the monoid $T$ is replaced by a group $G$ and one is interested in idempotent pure surjective relational morphisms $\tau: S \multimap G$ and their inverses $\tau^{-1}: G \rightarrow S$.

In the inverse case, the associated covers can be described in terms of prehomomorphisms from $S$ into the inverse monoid of all cosets of subgroups of $G$ [12]. For each $s \in S$, the subset $s \tau$ is a coset of $G$; in the left ample case, however, the subsets $s \tau$ of $T$ do not appear to enjoy any corresponding property, and so we do not have analogues of these particular results.

The surjective relational morphisms from $G$ to $S$ and their associated covers are described in [12] in terms of dual prehomomorphisms from $G$ to the inverse monoid of all permissible subsets of $S$. There are analogous results for the left ample case which we describe in the following sections.

\section{COMPATIBLE SUBSETS}

Let $S$ be a left ample semigroup. We construct a semigroup $C(S)$ and an embedding from $S$ into $C(S)$; the elements of $C(S)$ are certain subsets of $S$. As we shall see, $C(S)$ is a weakly left $E$-ample semigroup, and so we now explain what such a semigroup is.

Let $S$ be a semigroup, $E(S)$ its set of idempotents, and $E$ a subset of $E(S)$. Suppose that $E$ is a commutative subsemigroup of $S$ such that every element of $S$ has a left identity in $E$. Then the relation $\leqslant$ on $S$ defined by $a \leqslant b$ if and 
only if $a=e b$ for some $e \in E$ is a partial order on $S$ which extends the natural semilattice order on $E$. When $E=E(S)$, we refer to $\leqslant$ as the natural partial order on $S$.

We say that $S$ is left E-semiadequate if, for each $a \in S$, there is a minimum (in the semilattice order on $E$ ) left identity $a^{+}$of $a$ in $E$. It is easy to see that in such a semigroup, $a \leqslant b$ if and only if $a=a^{+} b$.

If $S$ is left $E$-semiadequate and also satisfies the following two conditions for all elements $a, b$ of $S$ and all idempotents $e$ in $E$, then it is weakly left E-ample:

(1) $(a b)^{+}=\left(a b^{+}\right)^{+}$

(2) $a e=(a e)^{+} a$.

An elementary property of weakly left $E$-ample semigroups, which will be used frequently without further mention, is given in the next lemma.

Lemma 2.1. Let $S$ be a weakly left E-ample semigroup. Then for all elements a of $S$ and idempotents $e$ in $E$,

$$
(e a)^{+}=e a^{+}
$$

For further information about weakly left $E$-ample semigroups see [8] and [6]. In particular, if $S$ is a left ample semigroup, then it follows from Lemma 1.1 that $a^{+}$is the minimum left identity of $a$ in $S$, and that as a $(2,1)$-algebra, $S$ is weakly left $E$-ample with $E=E(S)$.

Let $S$ be a left ample semigroup. We say that a nonempty subset $A$ of $S$ is compatible if it satisfies the following condition:

$(C)$ if $a, b \in A$, then $a^{+} b=b^{+} a$.

Note that condition $(C)$ is equivalent to the existence of a greatest lower bound $c$ of $a$ and $b$ with $c^{+}=a^{+} b^{+}$. For, if such a bound exists, then $c \leqslant a, b$ so that

$$
c=c^{+} a=c^{+} b=b^{+} a=a^{+} b .
$$

Conversely, if condition $(C)$ holds, then certainly $a^{+} b$ is a lower bound of $a$ and $b$, and if $d \leqslant a, b$, then $d=d^{+} a=d^{+} b$ so that $d=d^{+} a=d^{+} a^{+} a=d^{+} a^{+} b$, that is, $d \leqslant a^{+} b$. Hence $a^{+} b$ is the greatest lower bound of $a$ and $b$; also $\left(a^{+} b\right)^{+}=a^{+} b^{+}$ by Lemma 2.1 .

It follows from this observation and Lemma 1.11 of [9] that, in an inverse semigroup, a compatible subset (in our sense) is a subset in which any two elements are left compatible.

We now define $C(S)$ to be the set of all compatible order ideals of $S$. We caution the reader that our notation does not agree with that of inverse semigroup theory, and that the results we obtain are analogues of those in inverse semigroup theory rather than generalisations. We start by giving a weak analogue of a result of Schein [14] (see also [9]) on the semigroup structure of $C(S)$.

Observe that $E(S)$ is a member of $C(S)$. Indeed, any order ideal of $E(S)$ belongs to $C(S)$. Also, every principal order ideal of $S$ is in $C(S)$. For, let 
$[a]=\{b \in S: b \leqslant a\}$. Certainly, $[a]$ is an order ideal. If $b, c \in[a]$, then $b=e a$ and $c=f a$ for some idempotents $e, f$ so that

$$
b^{+} c=(e a)^{+} c=e a^{+} f a=f a^{+} e a=(f a)^{+} b=c^{+} b .
$$

The following easy lemma will be useful.

Lemma 2.2. Let $S$ be a left ample semigroup, and let $A$ be a compatible subset of $S$. If $a, a^{+} \in A$, then $a=a^{+}$.

Proof. By definition, $a=a^{+} a=\left(a^{+}\right)^{+} a=a^{+} a^{+}=a^{+}$.

Defining $\iota: S \rightarrow C(S)$ by $\iota(s)=[s]$, we give the main properties of $C(S)$ in the following result.

For any subset $A$ of $S$, let $A^{+}=\left\{a^{+}: a \in A\right\}$. Let $E$ be the set of order ideals of $E(S)$.

Proposition 2.3. Let $S$ be a left ample semigroup, and let $E$ be the set of all order ideals of $E(S)$. Then, under multiplication of subsets, $C(S)$ is a weakly left E-ample semigroup with left identity $E(S)$, the map $\iota: S \rightarrow C(S)$ is an injective +-homomorphism and, for every member $A$ of $C(S)$, there is an element $a \in S$ such that $\iota(a) \leqslant A$.

Proof. Let $A, B \in C(S)$. If $a \in A, b \in B$ and $c \leqslant a b$, then $c=c^{+} a b$. Now $c^{+} a \in A$ since $A$ is an order ideal, and so $c \in A B$.

To see that $A B$ is compatible, let $x=a b, y=c d \in A B$ where $a, c \in A$ and $b, d \in B$. Now $A E(S) \subseteq A$, because if $a \in A$ and $e \in E(S)$, then $a e=(a e)^{+} a \leqslant a$. Hence $a b^{+}, c d^{+} \in A$, and so

$$
x^{+} c d^{+}=(a b)^{+} c d^{+}=\left(a b^{+}\right)^{+} c d^{+}=\left(c d^{+}\right)^{+} a b^{+}=(c d)^{+} a b^{+}=y^{+} a b^{+} .
$$

Thus

$$
\begin{aligned}
x^{+} y & =x^{+} c d=x^{+} c d^{+} d=y^{+} a b^{+} d=y^{+} a d^{+} b \\
& =y^{+}\left(a d^{+}\right)^{+} a b=\left(a d^{+}\right)^{+} y^{+} x \\
& \leqslant y^{+} x .
\end{aligned}
$$

Similarly, $y^{+} x \leqslant x^{+} y$ and so $x^{+} y=y^{+} x$ as required. Thus $C(S)$ is closed and hence a semigroup.

That $E(S)$ is a left identity of $C(S)$ follows from the facts that $S$ is left ample, and the members of $C(S)$ are order ideals.

We know that $E \subseteq C(S)$, and clearly, members of $E$ are idempotents. If $A \in C(S)$, define $A^{+}$to be the set $\left\{a^{+}: a \in A\right\}$. Clearly, $A^{+} \subseteq E(S)$. If $a^{+} \in A^{+}$and $e \in E(S)$ with $e \leqslant a^{+}$, then $e=e a^{+}=(e a)^{+}$. As $e a \in A$, we have $e \in A^{+}$, and $A^{+}$is an order ideal of $E(S)$. Hence $A^{+} \in E$.

Clearly, $A^{+} A=A$. If $F \in E$ and $F A=A$, let $a \in A$. Then $a=f a$ for some $f \in F$ so that $a^{+}=(f a)^{+}=f a^{+}$. Hence $a^{+} \leqslant f$, and so $a^{+} \in F$. Thus $A^{+} \subseteq F$ and consequently $A^{+} \leqslant F$ in the natural partial order on $E$. 
Thus $C(S)$ is weakly left $E$-adequate.

We now show that the natural partial order on $C(S)$ coincides with inclusion. Let $A, B \in C(S)$. If $A \leqslant B$, then $A=F B$ for some $F \in E$, and so $A \subseteq B$ since $B$ is an order ideal of $S$. Now suppose that $A \subseteq B$. Then $A=A^{+} A \subseteq A^{+} B$. If $x \in A^{+} B$, then $x=a^{+} b$ for some $a \in A$ and $b \in B$. Since $a, b \in B$, we have $a^{+} b=b^{+} a \leqslant a$ so that $a^{+} b \in A$ and we have $A^{+} B=A$.

Next, we show that the two defining conditions for $C(S)$ to be weakly left ample hold. Let $A, B \in C(S)$. Since $\left(B A^{+}\right)^{+} B A=\left(B A^{+}\right)^{+} B A^{+} A=B A^{+} A=B A$, we have $(B A)^{+} \leqslant\left(B A^{+}\right)^{+}$. On the other hand, if $e \in\left(B A^{+}\right)^{+}$, then $e=\left(b a^{+}\right)^{+}$ for some $b \in B, a \in A$. As $S$ is left ample, $\left(b a^{+}\right)^{+}=(b a)^{+} \in(B A)^{+}$and consequently, $(B A)^{+}=\left(B A^{+}\right)^{+}$.

If $F \in E$, and $x \in A F$, then, for some $a \in A, f \in F$, we have $x=a f=$ $(a f)^{+} a \in(A F)^{+} A$ so that $A F \subseteq(A F)^{+} A$, that is, $A F \leqslant(A F)^{+} A$. Thus $A F=(A F)^{+}\left((A F)^{+} A\right)=(A F)^{+} A$.

It is easy to check that $\iota$ is an injective +-homomorphism; furthermore, if $A \in C(S)$ and $a \in A$, then clearly, $\iota(a) \leqslant A$.

\section{DUAL PREHOMOMORPHISMS AND COVERS}

Let $S$ and $T$ be left ample semigroups. A function $\theta: S \rightarrow T$ is a dual prehomomorphism if , for all $a, b \in S$,

$$
(a b) \theta \geqslant(a \theta)(b \theta) \text { and } a^{+} \theta \geqslant(a \theta)^{+} .
$$

Let $S$ be a left ample semigroup, and $T$ be a left cancellative monoid. In this section, we give conditions in terms of dual prehomomorphisms from $T$ into $C(S)$ for the existence of proper covers of $S$ over $T$, obtaining an analogue of [12, Theorem 4.5]. To be more precise, we prove the following theorem which is a consequence of Propositions 1.4, 3.2 and 3.3. Note that we identify $s \in S$ with its image $\iota(s)$ in $C(S)$.

Theorem 3.1. Let $\theta: T \rightarrow C(S)$ be a dual prehomomorphism such that, for each $s \in S$, there exists $t \in T$ with $s \leqslant t \theta$. Then $\{(t, s) \in T \times S: s \leqslant t \theta\}$ is a proper cover of $S$ over $T$. Conversely, every proper cover of $S$ over $T$ has this form for some dual prehomomorphism $\theta$ of $T$ into $C(S)$.

First, we show that dual prehomomorphisms give rise to proper covers.

Proposition 3.2. Let $S$ be a left ample +-subsemigroup of a weakly left Eample semigroup $C$ such that for every element $c \in C$ there exists $a \in S$ such that $a \leqslant c$. Let $T$ be a left cancellative monoid, and suppose that $\theta: T \rightarrow C$ is a dual prehomomorphism and define a relation $\tau$ between $T$ and $S$ by the rule

$$
t \tau=\{s \in S: s \leqslant t \theta\} .
$$

Then $\tau$ is a proper relational morphism, and it is surjective if and only if for each $s \in S$, there exists $t \in T$ such that $s \leqslant t \theta$. 
Proof. Certainly, $t \tau \neq \emptyset$ for every $t \in T$, and it is straightforward to verify that $\left(t_{1} \tau\right)\left(t_{2} \tau\right) \subseteq\left(t_{1} t_{2}\right) \tau$ for all $t_{1}, t_{2} \in T$.

If $a \in t \tau$, then $a \leqslant t \theta$, and so $a=a^{+}(t \theta)$. Hence

$$
a^{+}=\left(a^{+}(t \theta)\right)^{+}=a^{+}(t \theta)^{+} \leqslant(t \theta)^{+} \leqslant t^{+} \theta=1 \theta,
$$

so that $a^{+} \in t^{+} \tau$ and $(t \tau)^{+} \subseteq t^{+} \tau$.

Thus $\tau$ is a relational morphism. It is proper because, if $a, b \in t \tau$ for some $t \in T$, then $a=a^{+}(t \theta)$ and $b=b^{+}(t \theta)$ so that $b^{+} a=a^{+} b$ as required.

The assertion about surjectivity is clear.

We now show that there is a dual prehomomorphism associated with each proper cover of a left ample semigroup $S$ over a right cancellative monoid $T$ (or what amounts to the same thing, with each proper surjective relational morphism from $T$ to $S$ ).

Proposition 3.3. Let $S$ be a left ample semigroup, and $T$ be a left cancellative monoid. If $\tau: T \rightarrow S$ is a proper surjective relational morphism, then $t \tau$ is a compatible order ideal of $S$ for each $t$ in $T$, and the function $\theta: T \rightarrow C(S)$ defined by $t \theta=t \tau$ is a dual prehomomorphism with the property that, for each $s \in S$, there exists $t \in T$ with $s \leqslant t \theta$. Moreover,

$$
\operatorname{gr}(\tau)=\{(t, s) \in T \times S: s \leqslant t \theta\} .
$$

Proof. We claim that $1 \tau=E(S)$. For, if $a \in 1 \tau$, then also $a^{+} \in 1 \tau$, and, as $\tau$ is proper, we have $\left(a^{+}\right)^{+} a=a^{+} a^{+}$so that $a=a^{+} \in E(S)$. If $e \in E(S)$, then since $\tau$ is surjective, $e \in t \tau$ for some $t$, and so $e=e^{+} \in(t \tau)^{+} \subseteq t^{+} \tau=1 \tau$, proving the claim.

Now, if $a \in t \tau$ for some $t$ and $b \leqslant a$, then $b=b^{+} a \in(1 \tau)(t \tau) \subseteq t \tau$ so that $t \tau$ is an order ideal; it is compatible since $\tau$ is proper.

Thus $t \theta \in C(S)$ and it is clear that $\theta$ is a dual prehomomorphism. Further, since $\tau$ is surjective, $\theta$ has the claimed property by Proposition 3.2. The final assertion is also clear in view of the definition of $\theta$.

\section{FACTORISABle LeFt AMPle MONOIDS}

Dualising the notion of factorisable right ample monoid [2], we say that a left ample monoid $F$ is factorisable if $F=E T$ where $E=E(F)$ and $T$ is the $\mathscr{R}^{*}$-class of the identity. Note that $T$ is a right cancellative submonoid of $F$. We say that $F$ is $c$-factorisable if every element of $T$ is cancellable (in $F$ ). Observe that if $T$ is actually a group, then it must be the $\mathscr{H}$-class of the identity. In this case, an element of $F$ can be written as $e g$ for some $e \in E$ and unit $g$ of $F$; clearly, $g^{-1} e$ is an inverse of $e g$ and so $F$ is an inverse monoid. For emphasis, we record this observation in the following lemma.

Lemma 4.1. Let $F=E T$ be a factorisable right ample monoid. If $T$ is a group, then $F$ is inverse. 
A +-embedding $\theta$ of a left ample semigroup $S$ in a factorisable left ample monoid $F=E T$ is strict if for each element $t$ of $T$, there is an element $s \in S$ such that $s \theta \leqslant t$. We start by showing that, for any left ample semigroup $S$, such an embedding exists and that it gives rise to a proper cover of $S$ over $T$.

Lemma 4.2. If $S$ is a left ample semigroup, then there is a strict+-embedding of $S$ into a c-factorisable left ample monoid.

Proof. By Proposition 1.2 of [3], there is a +-embedding $\psi$ of $S$ into an inverse semigroup $I$. By [9, Theorem 2.2.3], $I$ can be embedded into a factorisable inverse monoid $J$; let $\varphi$ be this embedding. Then $\varphi$ is a +-embedding, and so $\theta=\psi \varphi$ is a +-embedding of $S$ into $J$.

Let $H$ be the group of units of $J$ and put

$$
T=\{t \in H: s \theta \leqslant t \text { for some } s \in S\} .
$$

Since the natural order is compatible, $T$ is a submonoid of $H$. Now put

$$
F=\{x \in J: x \leqslant t \text { for some } t \in T\} .
$$

Then $F$ is a full submonoid of $J$ and hence it is left ample. The elements of $T$ are the cancellable elements in $F$ so that $F$ is $c$-factorisable. Clearly, $S \theta \subseteq F$ and $\theta: S \rightarrow F$ is a strict +-embedding.

Proposition 4.3. Let $S$ be a left ample semigroup, and $\theta: S \rightarrow F=E T$ be a strict + -embedding of $S$ into a factorisable left ample monoid. If

$$
P=\{(s, t): s \theta \leqslant t\},
$$

then $P$ is a+-subsemigroup of $S \times T$, and is a proper cover of $S$ over $T$.

Proof. Define $\tau: S \multimap T$ by $s \tau=\{t \in T: s \theta \leqslant t\}$. Then $s \tau \neq \emptyset$ for all $s \in S$ since $F$ is factorisable. If $e \in E(S)$, then $e \theta \leqslant 1$, so $1 \in e \tau$. Hence $(s \tau)^{+}=\{1\} \subseteq s^{+} \tau$. Compatibility of the natural order gives $(a \tau)(b \tau) \subseteq(a b) \tau$ for $a, b \in S$ so that $\tau$ is a relational morphism. It is surjective since $\theta$ is a strict embedding.

Finally, if $t \in r \tau \cap s \tau$, then $r \theta \leqslant t$ and $s \theta \leqslant t$ so that $r \theta=\left(r^{+} \theta\right) t$ and $s \theta=\left(s^{+} \theta\right) t$. Hence

$$
\left(r^{+} s\right) \theta=\left(r^{+} \theta\right)(s \theta)=\left(r^{+} \theta\right)\left(s^{+} \theta\right) t=\left(r^{+} s^{+}\right) \theta t=\left(s^{+} r\right) \theta
$$

and so $r^{+} s=s^{+} r$.

Thus $\tau$ is a proper relational morphism, and so, by Proposition 1.4, $P$ is a proper cover of $S$ over $T$.

By virtue of Lemma 4.2 and Proposition 4.3, every left ample semigroup has a proper cover which arises from a strict embedding into a $c$-factorisable left ample monoid. McAlister and Reilly [12] (see also [9]) show that all $E$-unitary covers of an inverse semigroup arise from strict embeddings into factorisable inverse monoids, but as we shall see in the next section, the analogue for left ample semigroups does not hold. 


\section{E-DENSE LEFT AMPLE SEMigRoups}

We have been unable to determine necessary and sufficient conditions for a proper cover of a left ample semigroup to be (isomorphic to) a proper cover which arises from a strict embedding. We now consider the question for $E$-dense proper covers of $E$-dense left ample semigroups.

Recall that a semigroup $S$ is $E$-dense if for all $a \in S$, there is an element $b \in S$ such that $b a$ is idempotent. From [13], we have

Lemma 5.1. The following conditions are equivalent for a semigroup $S$ with set of idempotents $E(S)$.

(1) $S$ is E-dense;

(2) for all $a \in S$, there is an element $c \in S$ such that ac $\in E(S)$;

(3) for all $a \in S$, there is an element $d \in S$ such that ad, $d a \in E(S)$;

(4) for all $a \in S$, there is an element $x \in S$ such that $x a x=x$.

For $E$-dense left ample semigroups, we have a stronger version of Lemma 4.2.

Proposition 5.2. If $S$ is an E-dense left ample semigroup, then there is a strict + -embedding of $S$ into a factorisable inverse monoid.

Proof. We use the strict +-embedding $\theta: S \rightarrow F$ of Lemma 4.2. Since $F$ is a $c$-factorisable left ample monoid, it is enough to show that it is inverse, and, by Lemma 4.1, this follows if we show that $T$ (the set of cancellable elements of $F$ ) is a subgroup of the group $H$ of units of $J$.

Let $t \in T$ so that $s \theta \leqslant t$ for some $s \in S$. Let $r \in S$ be such that $s r$ is idempotent, and let $u \in T$ be such that $r \theta \leqslant u$. Then $s \theta=e t$ and $r \theta=f u$ for some idempotents $e, f$ in $F$. Since $F$ is left ample, we have

$$
(\text { etf })^{+} \text {etu }=\text { etf } u=(s \theta)(r \theta)=(s r) \theta
$$

so that $(s r) \theta \leqslant t u$. Since $s r$ is idempotent, $(s r) \theta=(s r) \theta t u$ and so $(s r) \theta(t u)^{-1}=$ $(s r) \theta$ where $(t u)^{-1}$ is the inverse of $t u$ in $H$. But $(s r) \theta \leqslant(t u)^{-1}$ implies $(t u)^{-1} \in T$ and so $t^{-1}=u(t u)^{-1} \in T$ and $T$ is a subgroup of $H$.

Let $S$ be a left ample semigroup, and $\theta: S \rightarrow F=E T$ be a strict +-embedding of $S$ into a factorisable left ample monoid. We now show that if the proper cover $P$ arising from the embedding is $E$-dense, then $F$ is inverse.

Lemma 5.3. If $P$ is E-dense, then $F$ is inverse.

Proof. By [5, Lemma 1.6], $P / \sigma$ is a group, but it follows from Propositions 1.4 and 4.3 that $P / \sigma \cong T$, so by Lemma $4.1, F$ is inverse. 
Let $S$ be an $E$-dense left ample semigroup, and let $M$ be a proper $E$-dense left ample cover of $S$ over a group $G$ with surjective homomorphisms $\alpha$ and $\beta$ :

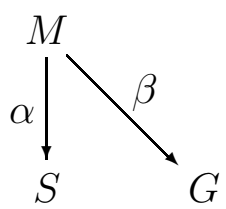

with $\left.\alpha\right|_{E(M)}$ an isomorphism from $E(M)$ onto $E(S), \beta \beta^{-1}=\sigma$ and $\tau=\alpha^{-1} \beta$. We say that $M$ is an even cover of $S$ over $G$ if for all $s \in S$,

$$
g, h \in s \tau \text { implies } g h^{-1} \in s^{+} \tau \text {. }
$$

Note that the defining condition is equivalent to asserting that $e \tau$ is a subgroup for every idempotent in $S$ and that $s \tau$ is contained in a right coset of $s^{+} \tau$ for every $s \in S$. First, we show that a proper cover constructed from a strict embedding into a factorisable inverse monoid is an even cover.

Proposition 5.4. Let $\theta: S \rightarrow F$ be a strict embedding of an E-dense left ample semigroup $S$ into a factorisable inverse monoid $F$, and let $P$ be the proper cover arising from the embedding. Then $P$ is E-dense and even. Moreover, if $S$ is inverse, so is $P$.

Proof. Since $P / \sigma \cong G$ is a group, it follows from Lemma 1.6 of [5] that $P$ is E-dense.

If $g, h \in s \tau$, then $s \theta \leqslant g, h$ so that $s \theta=x g=y h$ for some idempotents $x, y \in F$. In fact, $x=y=(s \theta)^{+}=s^{+} \theta$. Hence $\left(s^{+} \theta\right) g h^{-1}=s^{+} \theta$, that is, $s^{+} \theta \leqslant g h^{-1}$ and so $g h^{-1} \in s^{+} \tau$.

If $S$ is inverse, the construction is that of McAlister and Reilly, and so by [12, Proposition 1.3], $P$ is inverse.

Now let $M$ be any $E$-dense even cover of $S$ over a group $G$. Our aim is to show that $M$ arises from a strict embedding into a factorisable inverse monoid. Our proof is inspired by that of McAlister and Reilly for [12, Theorem 1.7] although there are considerable differences in detail.

Put $E=E(S)$, and define a relation $\sim$ on $E \times G$ by

$$
(e, g) \sim(f, h) \text { if and only if } e=f \text { and } g h^{-1} \in e \tau .
$$

Note that $\sim$ is an equivalence relation since $e \tau$ is a subgroup for each $e \in E$.

We know that $\alpha$ maps $E(M)$ isomorphically onto $E$; for $e \in E$, we write $\bar{e}$ for the idempotent in $M$ such that $\bar{e} \alpha=e$. Let $s \in S$ and $(e, g) \in E \times G$. We say that $(e, g)$ is $s$-accessible if there are elements $n, m \in M$ such that $m \alpha=s$, $n \beta=g$ and $\bar{e}=n^{+}=(n m)^{+}$. Note that $n$ is uniquely determined by these conditions since $M$ is proper. Also, if $p \in s \alpha^{-1}$, then

$$
\left.(n p)^{+} \alpha=((n p) \alpha)^{+}=((n m)) \alpha\right)^{+}=(n m)^{+} \alpha
$$

so that $(n p)^{+}=(n m)^{+}$, and hence the $s$-accessibility of $(e, g)$ is independent of the choice of $m$. 
Next, we note that for any $s \in S$, the pair $\left(s^{+}, 1\right)$ is $s$-accessible. For the defining condition, we can take $n=m=\overline{s^{+}}$.

Now suppose that $(e, g)$ is $s$-accessible and that $(e, g) \sim(e, h)$. Then $h g^{-1} \in e \tau$, and there are elements $m, n \in M$ with $m \alpha=s$ and $\bar{e}=n^{+}=(n m)^{+}$, and $n \beta=g$. Let $y \in M$ be such that $y \alpha=e$ and $y \beta=h g^{-1}$. Then $(y n) \beta=h g^{-1} g=h$. Also $(y n) \alpha=(y \alpha)(n \alpha)=\left(n^{+} \alpha\right)(n \alpha)=n \alpha$, so $(y n)^{+} \alpha=n^{+} \alpha$ whence $(y n)^{+}=n^{+}$. Similarly, $(y n m)^{+}=(n m)^{+}$. Hence the $\sim$-equivalence class of $(e, g)$, which we denote by $[e, g]$, consists of $s$-accessible pairs.

Now let

$$
Z=(E \times G) / \sim \sqcup G
$$

be the disjoint union of the set of $\sim$-equivalence classes and $G$. For each $s \in S$, define a subset $U_{s}$ of $(E \times G) / \sim$ by

$$
U_{s}=\{[e, g]:(e, g) \text { is } s \text {-accessible }\} .
$$

Let $g \in s \tau$. Define a partial function $\theta_{s}$ on $Z$ with domain $U_{s}$ by

$$
[e, u] \theta_{s}=[e, u g] \text {. }
$$

To see that $\theta_{s}$ is well defined, let $[e, u]=[e, v] \in U_{s}$ and $g, h \in s \tau$. Then $u v^{-1} \in e \tau$ so that there is an element $y \in M$ with $y \alpha=e$ and $y \beta=v u^{-1}$. Since $(e, u)$ is $s$-accessible, there is an element $n \in M$ with $n \beta=u$ and $\bar{e}=n^{+}=(n m)^{+}$ for all $m \in s \alpha^{-1}$. Let $p, q \in s \alpha^{-1}$ be such that $p \beta=g$ and $q \beta=h$.

Then $(n p) \beta=u g$ and $(y n q) \beta=v u^{-1} u h=v h$. Also

$$
(y n q) \alpha=(y \alpha)(n \alpha) s=\left(n^{+} \alpha\right)(n \alpha) s=(n \alpha) s=(n p) \alpha,
$$

and hence $u g, v h \in((n \alpha) s) \tau$. Since $M$ is even, $(u g)(v h)^{-1} \in((n \alpha) s)^{+} \tau$. But, $((n \alpha) s)^{+}=\left((n p)^{+}\right) \alpha=(n p)^{+} \alpha=e$. Thus $(e, u g) \sim(e, v h)$, and $\theta_{s}$ is well defined.

We claim that $\theta_{s}$ is one-one. Suppose that $[e, u],[f, v] \in U_{s}$ and that $[e, u] \theta_{s}=$ $[f, v] \theta_{s}$. Then $[e, u g]=[f, v g]$ so that $e=f$ and $u v^{-1}=u g(v g)^{-1} \in e \tau$ whence $[e, u]=[f, v]$.

Let $I_{Z}$ be the symmetric inverse monoid on $Z$.

Lemma 5.5. The mapping $\theta: S \rightarrow I_{Z}$ given by $s \theta=\theta_{s}$ is $a+$-embedding.

Proof. First, for elements $r, s \in S$, we show that $\operatorname{Dom} \theta_{r} \theta_{s}=\operatorname{Dom} \theta_{r s}=U_{r s}$. If $[e, u] \in \operatorname{Dom} \theta_{r} \theta_{s}$, then certainly $[e, u] \in U_{r}$ and $[e, u g] \in U_{s}$ for $g \in r \tau$. Hence if $m \in r \alpha^{-1}$ with $m \beta=g$, and $n \in s \alpha^{-1}$, there exist $m_{1}, m_{2} \in M$ such that

$$
\bar{e}=m_{1}^{+}=\left(m_{1} m\right)^{+}=m_{2}^{+}=\left(m_{2} n\right)^{+}
$$

and $m_{1} \beta=u$ and $m_{2} \beta=u g=u(m \beta)$. Now $\left(m_{1} m\right) \beta=m_{2} \beta$ so that $\left(m_{1} m\right) \sigma m_{2}$, and since $\left(m_{1} m\right)^{+}=m_{2}^{+}$and $M$ is proper, we have $m_{1} m=m_{2}$.

Since $\left(\left(m_{1} m\right) n\right)^{+}=m_{1}^{+}=\bar{e}$ and $(m n) \alpha=r s$, it follows that $[e, u] \in U_{r s}$.

On the other hand, if $[e, u] \in U_{r s}$, then there is an element $p$ in $M$ such that $\bar{e}=p^{+}=(p m n)^{+}$and $p \beta=u$. By Lemma 1.1, $p^{+}=(p m)^{+}$and so $[e, u] \in U_{r}$. Moreover, $[e, u] \theta_{r}=[e, u(m \beta)]$, so that since $\bar{e}=(p m)^{+}=((p m) n)^{+}$ 
and $(p m) \beta=u(m \beta)$, we have $[e, u(m \beta)] \in U_{s}$ whence $[e, u] \in \operatorname{Dom} \theta_{r} \theta_{s}$ as required.

For $[e, u] \in U_{r s}$, we have

$$
[e, u] \theta_{r} \theta_{s}=[e, u(m \beta)(n \beta)]=[e, u(m n) \beta]=[e, u] \theta_{r s}
$$

so that $\theta$ is a semigroup homomorphism.

Now $\theta_{s}^{+}=\theta_{s} \theta_{s}^{-1}$ so that $\theta_{s}^{+}$is the identity map with domain $U_{s}$. It is easy to see that $\theta_{s^{+}}$has the same domain and acts in the same way so that $s^{+} \theta=(s \theta)^{+}$.

Finally, if $\theta_{r}=\theta_{s}$, and $m \in r \alpha^{-1}, n \in s \alpha^{-1}$, then, putting $f=m^{+} \alpha$, we have

$$
[f, m \beta]=[f, 1] \theta_{r}=[f, 1] \theta_{s}=[f, n \beta] .
$$

Thus $(m \beta)(n \beta)^{-1} \in f \tau$ so that, in particular, $m \beta, n \beta \in a \tau$ for some $a \in S$, and

$$
r=m \alpha=a=n \alpha=s .
$$

Hence $\theta$ is a + -embedding.

We also want to embed $G$ in the group of units if $I_{Z}$. For $g \in G$, we define $\pi_{g}: Z \rightarrow Z$ by the rule

$$
[e, u] \pi_{g}=[e, u g] \text { and } u \pi_{g}=u g \text { for all }(e, u) \in E \times G \text { and } u \in G \text {. }
$$

It is easy to see that $\pi_{g}$ is well defined and one-one. Moreover, $\pi_{g} \pi_{g^{-1}}=\mathrm{id}_{Z}=$ $\pi_{g^{-1}} \pi_{g}$ so that $\pi_{g}$ is a unit of $I_{Z}$. Clearly, $\pi: G \rightarrow I_{Z}$ given by $g \pi=\pi_{g}$ is an embedding.

Proposition 5.6. Let $F=\left\{\gamma \in I_{Z}: \gamma \leqslant \pi_{g}\right.$ for some $\left.g \in G\right\}$. Then $F$ is a factorisable inverse monoid, $S \theta \subseteq F$ and $S$ is strictly embedded in $F$ by $\theta$. Further, $M$ is isomorphic to $P$ where

$$
P=\left\{\left(s, \pi_{g}\right) \in S \times G \pi: s \theta \leqslant \pi_{g}\right\} .
$$

Proof. Clearly, $F$ is a submonoid of $I_{Z}, G \pi$ is its group of units and $F=E(F) G \pi$, so that $F$ is a factorisable inverse monoid.

If $s \in S$, then, for $g \in s \tau$, we have $\theta_{s}=\operatorname{id}_{D} \pi_{g}$ where $D=U_{s}$, and so $S \theta \subseteq F$. Moreover, for each $g \in G$, there is some $s \in S$ with $g \in s \tau$, so the embedding $\theta$ (into $F$ ) is strict. Thus, as in Proposition 4.3,

$$
P=\left\{\left(s, \pi_{g}\right) \in S \times G \pi: s \theta \leqslant \pi_{g}\right\}
$$

is a proper cover of $S$ over $G$.

We identify $G$ with $G \pi$ and write the elements of $P$ as $(s, g)$. Now define $\varphi: M \rightarrow P$ by

$$
m \varphi=(m \alpha, m \beta) .
$$

Clearly, $\varphi$ is a homomorphism, and it is injective since $M$ is proper.

To see that $\varphi$ is onto, let $(s, g) \in P$. Then $s \theta \leqslant \pi_{g}$ and $s=m \alpha$ for some $m \in M$. Hence we have

$$
\left[s^{+}, m \beta\right]=\left[s^{+}, 1\right] \theta_{s}=\left[s^{+}, 1\right] \pi_{g}=\left[s^{+}, g\right]
$$


so that $g(m \beta)^{-1} \in s^{+} \tau$. Now $m \beta \in s \tau$, so

$$
g=g(m \beta)^{-1}(m \beta) \in\left(s^{+} \tau\right)(s \tau) \subseteq s \tau .
$$

Thus there is an element $q \in M$ with $q \alpha=s$ and $q \beta=g$, that is, $q \varphi=(s, g)$, and $\operatorname{so} \varphi$ is onto.

The following theorem is now an immediate consequence of the foregoing.

Theorem 5.7. Let $S$ be an E-dense left ample semigroup. Every even cover of $S$ over a group $G$ is isomorphic to one constructed from a strict embedding of $S$ into a factorisable inverse monoid with group of units isomorphic to $G$.

Corollary 5.8. Let $S$ be an inverse semigroup. Every even cover of $S$ over a group is inverse.

Proof. Let $P$ be an even cover of $S$ over a group. By Theorem 5.7, $P$ can be regarded as a proper cover constructed from a strict embedding of $S$ into a factorisable inverse monoid. Hence, as noted in Proposition 5.4, $P$ is inverse.

We conclude with an example of an $E$-dense proper cover which is not even.

Example. Let $G=\langle g\rangle$ be an infinite cyclic group with identity $e$, and let $A=$ $\left\{a^{k}: k \geqslant 0\right\}$ be an infinite cyclic monoid with identity $1=a^{0}$. Extend the multiplications of $G$ and $A$ to $M=A \cup G$ by defining $a^{i} g^{j}=g^{j} a^{i}=g^{i+j}$ for all integers $i, j$ with $i \geqslant 0$. Clearly, $M$ is a commutative $E$-dense proper left ample monoid.

Let $H=\langle h\rangle$ be a finite cyclic group, and $S$ be the semigroup $H \cup\{0\}$. The mapping which sends $a^{i}$ to $h^{i}$ and $g^{j}$ to 0 makes $M$ into a proper cover of $S$, but $M$ is not inverse, and so, by Corollary 5.8 , it cannot be even.

The example shows that not every proper cover of a left ample semigroup arises from a strict embedding into a factorisable left ample monoid. For, if $M$ did so arise, say from a strict embedding $\theta: S \rightarrow F$, then by Lemma 5.3, $F$ must be inverse. But, by Proposition 5.4, this implies that $M$ is inverse, a contradiction.

\section{REFERENCES}

[1] K. Auinger and P.G. Trotter, A syntactic approach to covers for $E$-dense semigroups over group varieties, Mathematika, 48 (2001), 231-245.

[2] A. El Qallali, Factorisable right adequate semigroups, Proc. Edinburgh Math. Soc., 24 (1981), 171-178.

[3] J. Fountain, A class of right PP monoids, Quart. J. Math. Oxford., 28 (1977), 285-300.

[4] J. Fountain, Adequate semigroups, Proc. Edinburgh Math. Soc., 22 (1979), 113-125.

[5] J. Fountain and G.M.S. Gomes, Left proper E-dense monoids, J. Pure Appl. Alg., 80 (1992), 1-27.

[6] V. Gould, (Weakly) left E-ample semigroups, http://www-users.york.ac.uk/ varg1

[7] J.M. Howie, Fundamentals of semigroup theory, Oxford University Press, 1995.

[8] M.V. Lawson, Semigroups and ordered categories I. The reduced case, J. Algebra, 141 (1991), 422-462. 
[9] M.V. Lawson, Inverse semigroups, World Scientific, 1998.

[10] D.B. McAlister, Groups, semilattices and inverse semigroups, Trans. Amer. Math. Soc., 192 (1974), 227-244.

[11] D.B. McAlister, Groups, semilattices and inverse semigroups II, Trans. Amer. Math. Soc., 196 (1974), 351-370.

[12] D.B. McAlister and N.R. Reilly, E-unitary covers for inverse semigroups, Pacific J. Math. 68 (1977), 161-174.

[13] H. Mitsch, Subdirect products of E-inversive semigroups, J. Austral. Math. Soc. 48 (1990), 66-78.

[14] B.M. Schein, Completions, translational hulls, and ideal extensions of inverse semigroups, Czechoslovak J. Math. 23 (1973), 575-610. 